\title{
Fixing the renormalisation scheme in NNLO perturbative QCD using conformal limit arguments
}

\author{
J. Rathsman* \\ Dept. of Radiation Sciences, Uppsala University, Box 535, S-75121 Uppsala, Sweden
}

\begin{abstract}
We discuss how the renormalisation scheme ambiguities in QCD can be fixed, when two observables are related, by requiring the coefficients in the perturbative expansion relating the two observables to have their conformal limit values, i.e. to be independent of the $\beta$-function of the renormalised coupling. We show how the next-to-leading order BLM automatic scale fixing method can be extended to next-to-next-to-leading order to fix both the renormalisation scale and $\beta_{2}$ in a unique way. As an example we apply the method to the relation between Bjorken's sum rule and $R_{e+e-}$ and compare with experimental data as well as other scheme fixing methods.
\end{abstract}

*rathsman@tsl.uu.se 


\section{INTRODUCTION}

In perturbative $\mathrm{QCD}$, observables are given by expansions in the strong coupling $\alpha_{s}$,

$$
R=\left(\frac{\alpha_{s}}{\pi}\right)^{N}\left[R_{0}+R_{1} \frac{\alpha_{s}}{\pi}+R_{2}\left(\frac{\alpha_{s}}{\pi}\right)^{2}+\ldots\right],
$$

where the coefficients $R_{i}$ can be calculated from the appropriate Feynman diagrams. The individual terms in the series depends on the renormalisation scheme one is using but the sum of the entire series is independent of the scheme according to the renormalisation group equation. However, when the series is truncated the result becomes renormalisation scheme dependent. This dependence is formally of higher order than the terms calculated in the series but numerically the difference between different schemes can be large. These differences give a theoretical uncertainty which in principle makes it impossible to make any absolute predictions since any result can be obtained by a finite renormalisation. By going to higher order in perturbation theory the renormalisation scheme dependence becomes smaller but in principle the problem remains. One can argue that it is only bad scheme choices that give 'crazy' results and that as long as one uses a 'sensible' scheme the result will also be 'sensible'. The question then arises, what is a 'sensible' scheme?

The question of how to choose an appropriate renormalisation scheme in QCD has been discussed many times. Three well-known methods for choosing the renormalisation scheme are the 'Effective Charge Scheme' by Grunberg [1], the 'Principle of Minimum Sensitivity' by Stevenson [2] and 'Automatic Scale Fixing' (BLM) by Brodsky, Lepage and Mackenzie [3]. All these methods are based on some more or less intuitive principle or set of arguments for how a perturbative series should behave.

Of special interest here is the BLM method which fixes the scale in next-to-leading order (NLO) using conformal limit arguments. In a conformally invariant theory the coupling $a=\alpha(\mu) / \pi$ is scale invariant, i.e.

$$
\frac{d a}{d \ln \mu}=\beta(a)=-\beta_{0} a^{2}-\beta_{1} a^{3}-\beta_{2} a^{4}-\ldots=0 .
$$

It is therefore natural to define the conformal limit of perturbative QCD as the limit $\beta_{i} \rightarrow 0$ [3:4]. This means that the coefficients $R_{i}$ in the perturbative series have their conformal limit values if they do not contain any explicit dependence on the $\beta$-function. For example, in NLO the perturbative coefficients should have no explicit $\beta_{0}$-dependence. In the BLM method this is achieved by absorbing all $\beta_{0}$-dependent NLO terms $\left(\beta_{0}=\frac{11}{2}-\frac{1}{3} N_{f}\right.$ where $N_{f}=$ number of active quark flavours) into the running of $\alpha_{s}$ by a suitable redefinition of the renormalisation scale. It should be noted that the renormalisation scale obtained by the BLM method can also be interpreted as the mean value of the virtualities in the gluon propagators [3, 5, 4, 66, :7].

A useful concept when discussing renormalisation scheme uncertainties is the effective charge [1] of an observable which contains all QCD-corrections. For example, the effective charge $\hat{a}_{R}$ of $R_{e^{+} e^{-}}$is defined by,

$$
R_{e^{+} e^{-}}\left(Q_{R}\right)=\frac{\sigma\left(e^{+} e^{-} \rightarrow \text { hadrons }\right)}{\sigma\left(e^{+} e^{-} \rightarrow \mu^{+} \mu^{-}\right)}=
$$




$$
=3 \sum_{i=1}^{N_{f}} e_{i}^{2}\left(1+\frac{3}{4} C_{F} \hat{a}_{R}\left(Q_{R}\right)\right) .
$$

Each effective charge has its own $\beta$-function [1] connected to it,

$$
\frac{d \hat{a}_{R}}{d \ln Q_{R}}=\hat{\beta}_{R}\left(\hat{a}_{R}\right)=-\beta_{0} \hat{a}_{R}^{2}-\beta_{1} \hat{a}_{R}^{3}-\hat{\beta}_{2, R} \hat{a}_{R}^{4}-\ldots
$$

where $\beta_{0}$ and $\beta_{1}$ are renormalisation scheme independent and $\hat{\beta}_{i, R}, i \geq 2$ are renormalisation scheme invariants. Thus, for each physical observable $A$ there is a specific $\hat{\beta}_{2, A}$ connected to it which is an inherent property of the effective charge. The perturbative series for an effective charge depends on the renormalisation scheme even in the conformally invariant theory but when two effective charges are related, one gets a relation that is independent of the intermediate scheme that was used.

In this paper we present a new generalisation of the BLM method to next-to-next-toleading order (NNLO) using the conformal limit arguments as starting point which fixes both the renormalisation scale and $\beta_{2}$ when two physical observables are related. The value for $\beta_{2}$ that is obtained is an intermediate value between the $\hat{\beta}_{2}$ 's of the two effective charges. (A generalisation to the factorisation scheme problem will be considered in a separate paper [8]). This is a variation of an approach by Grunberg and Kataev [9], but whereas they claimed that the prescription for making the coefficients $N_{f}$-independent is ambiguous, we will show that once the initial renormalisation scheme is fixed by relating two physical observables, the conformal limit arguments fixes the scheme in a unique way. We also compare with the single [10] and multi-scale extensions [4,99:11] of the BLM method to NNLO which fixes the renormalisation scale when two effective charges $A$ and $B$ are related, $\hat{a}_{A}=$ $\hat{a}_{B}\left(1+r_{1, A / B} \hat{a}_{B}+\ldots\right)$, using $\hat{\beta}_{2, B}$. As an example the conformal limit scheme fixing method is applied to the relation between Bjorken's sum rule in polarised deep inelastic scattering and $R_{e+e_{-}}$. The result is compared with a recently reported experimental determination of Bjorken's sum rule and the general renormalisation scheme dependence.

\section{THE CONFORMAL LIMIT SCHEME FIXING METHOD}

Consider an observable in NNLO depending on one energy scale $Q$ such as $R_{e^{+} e^{-}}\left(Q_{R}\right)$ defined by Eq. (3). The effective charge $\hat{a}_{R}$ contains all QCD-corrections,

$$
\begin{aligned}
\hat{a}_{R}\left(Q_{R}\right)=a\left(\mu, \beta_{2}, \ldots\right)[ & 1+r_{1}\left(Q_{R}, \mu\right) a\left(\mu, \beta_{2}, \ldots\right)+ \\
& \left.+r_{2}\left(Q_{R}, \mu, \beta_{2}\right) a^{2}\left(\mu, \beta_{2}, \ldots\right)\right],
\end{aligned}
$$

where the coefficients $r_{i}$ can be calculated using perturbative QCD. The renormalisation scheme dependence can be parametrised through the renormalisation scale $\mu$ and the coefficients in the $\beta$-function, $\beta_{i}$ for $i \geq 2$ [2]. Strictly speaking it is the ratio, $\mu / \Lambda$, of the renormalisation scale and the QCD scale parameter $\Lambda$ that is the relevant parameter but in the following we will often make the implicit assumption that $\Lambda$ is held fixed when $\mu$ is varied. This can be done by choosing a measurement of an effective charge to define $\Lambda$ as will be shown later. 
The first two terms in the renormalisation group equation for the coupling $a=\alpha_{s}(\mu) / \pi$,

$$
\frac{d a}{d \ln \mu}=\beta(a)=-\beta_{0} a^{2}-\beta_{1} a^{3}-\beta_{2} a^{4}-\ldots,
$$

are renormalisation scheme independent,

$$
\begin{aligned}
& \beta_{0}=\frac{11}{6} N_{C}-\frac{1}{3} N_{f}, \\
& \beta_{1}=\frac{17}{12} N_{C}^{2}-\frac{5}{12} N_{C} N_{f}-\frac{1}{4} C_{F} N_{f},
\end{aligned}
$$

whereas the higher order terms depend on the renormalisation scheme.

Applying self-consistency for the perturbative expansion of the effective charge with respect to the renormalisation scheme parameters,

$$
\frac{d \hat{a}_{R}}{d \ln \mu}, \frac{d \hat{a}_{R}}{d \beta_{2}}=\mathcal{O}\left(a^{4}\right),
$$

gives [2] the following renormalisation scheme invariants,

$$
\begin{aligned}
\hat{r}_{1} & =r_{1}-\beta_{0} \ln \frac{\mu}{\Lambda} \\
\hat{\beta}_{2, R} & =\beta_{2}-\beta_{1} r_{1}-\beta_{0} r_{1}^{2}+\beta_{0} r_{2},
\end{aligned}
$$

where $\hat{\beta}_{2, R}$ is the coefficient in the renormalisation group equation for the effective charge given by Eq. (田). In passing we also note that the expression for the renormalisation scheme invariant $\hat{r}_{1}$ shows explicitly that it is $\mu / \Lambda$ that is the relevant parameter for parametrising the renormalisation scheme dependence.

From the self-consistency requirements we also get the explicit $\mu$ - and $\beta_{j}$-dependence of the coefficients $r_{i}$,

$$
\begin{aligned}
r_{1}= & r_{1}^{*}+\beta_{0}\left[d^{*}+\ln \frac{\mu}{Q}\right], \\
r_{2}= & r_{2}^{*}-\frac{\beta_{2}-\beta_{2, \overline{M S}}}{\beta_{0}}+\beta_{1}\left[d^{*}+\ln \frac{\mu}{Q}\right]+ \\
& +\beta_{0}\left[e^{*}+2 r_{1} \ln \frac{\mu}{Q}\right]+\beta_{0}^{2}\left[f^{*}-\ln ^{2} \frac{\mu}{Q}\right],
\end{aligned}
$$

where we have assumed that the coefficients have been calculated in the $\overline{M S}$ scheme with $\mu=Q$ to fix the integration constants. (The ${ }^{*}$ is used to indicate terms that are independent of $\beta_{0}$ and $\beta_{1}$.) We also assume that $r_{1}$ and $r_{2}$ only contain $\beta_{0^{-}}$and $\beta_{1}$-dependent terms from loop-insertions which is why the $\beta_{0}$ term in $r_{1}$ and the $\beta_{1}$ term in $r_{2}$ are the same, i.e they are both given by $d^{*}$. This way we also fix the redundancy in how to divide $r_{2}$ into $\beta_{0}$ and $\beta_{1}$ dependent parts.

We are now in the position to apply the conformal limit arguments to the effective charge $\hat{a}_{R}$ to fix the renormalisation scheme parameters $\mu$ and $\beta_{2}$. First the renormalisation scale is 
fixed by requiring $r_{1}$ to be $\beta_{0}$-independent. From Eq. (11) we see that this can be obtained by choosing the renormalisation scale as

$$
\mu^{*}=\mu_{B L M}=Q \exp \left(-d^{*}\right)
$$

We also note that the renormalisation scale obtained in this way is the same as in the original BLM method.

Next $\beta_{2}$ is fixed by requiring $r_{2}$ to be $\beta_{0^{-}}$and $\beta_{1}$-independent, i.e. $r_{2}=r_{2}^{*}$. Using the renormalisation scheme invariant $\hat{\beta}_{2, R}$ we get the following expression for $r_{2}$,

$$
r_{2}=\left(r_{1}^{*}\right)^{2}+\frac{\beta_{1}}{\beta_{0}} r_{1}^{*}+\frac{\hat{\beta}_{2, R}-\beta_{2}}{\beta_{0}} .
$$

From this we see that by choosing a renormalisation scheme where $\beta_{2}$ is given by,

$$
\beta_{2}^{*}=\hat{\beta}_{2, R}+\beta_{1} r_{1}^{*}+\beta_{0}\left(r_{1}^{*}\right)^{2}-\beta_{0} r_{2}^{*},
$$

we get $r_{2}=r_{2}^{*}$. Note that this value of $\beta_{2}$ in general is different both from the effective charge value, $\beta_{2, R}$ and from $\beta_{2, \overline{M S}}$ which was used in the calculation. However, if $r_{i}^{*}=0$ then $\beta_{2}^{*}=\hat{\beta}_{2, R}$ and if $r_{i}^{*}=r_{i}$ then $\beta_{2}^{*}=\beta_{2, \overline{M S}}$.

This fixes the renormalisation scheme in NNLO up to the question of initial scheme, which is resolved when two physical observables are related as shown below. This does not introduce any new uncertainties since only relations between observables can be predicted in a renormalised theory and for each pair of observables we get a unique relation. The situation here is not different from what happens in the BLM method and its earlier extensions where it is also necessary to fix the initial renormalisation scheme to get a unique result. In [9] it was argued that 'in QCD, setting $r_{i}=r_{i}^{*}$ is always possible, but leaves us with an ambiguous prescription'. However, as we have shown above, there are no ambiguities once the initial renormalisation scheme has been fixed and this can be done using a physical observable as shown below.

The perturbative series for the effective charge $\hat{a}_{R}$ in NNLO thus becomes

$$
\hat{a}_{R}=a^{*}\left(1+r_{1}^{*} a^{*}+r_{2}^{*}\left(a^{*}\right)^{2}\right)
$$

where the $r_{i}^{*}$ 's contain no explicit $\beta_{j}$-terms. In this way we obtain the required feature that all signs of scale breaking, i.e. $\beta \neq 0$, is confined into the running of the coupling and the coefficients in the perturbative series have their conformal limit values. Finally $a^{*}$ can be obtained by solving the renormalisation group equation (6) with the fixed $\beta_{2}^{*}$.

Before ending this section we note that the method for fixing $\beta_{2}$ can be generalised to arbitrary order, $n \geq 2$. For this we need the renormalisation scheme invariants $\hat{\beta}_{n, R}$ in the renormalisation group equation for the effective charge Eq. (四). The general form for $\hat{\beta}_{n, R}$ is given in [1] and can be rewritten as,

$$
r_{n}=\sum_{j=0}^{n} c_{j, n} r_{1}^{n}+\frac{1}{n-1} \frac{\hat{\beta}_{n, R}-\beta_{n}}{\beta_{0}}
$$


where $c_{j, n}$ only depends on $\left\{\hat{\beta}_{i, R}, \beta_{i}\right\}$ with $i \leq n-1$. In previous steps of applying the conformal limit arguments, the renormalisation scale has been fixed so that $r_{1}=r_{1}^{*}$ and the $\beta_{i}$ 's, $(2 \leq i \leq n-1)$ have been fixed to $\beta_{i}=\beta_{i}^{*}$. So by requiring $r_{n}=r_{n}^{*}$ to contain no explicit $\beta_{i}$-terms for $i \leq n-1$ the value of $\beta_{n}$ is fixed to be,

$$
\beta_{n}^{*}=\hat{\beta}_{n, R}-(n-1) \beta_{0} r_{n}^{*}+(n-1) \beta_{0} \sum_{j=0}^{n} c_{j, n}^{*}\left(r_{1}^{*}\right)^{n}
$$

which is a generalisation of Eq. (15) to arbitrary $n \geq 2$.

\section{COMPARISON WITH OTHER SCALE FIXING METHODS}

In previous multi-scale extensions of the BLM method (denoted MBLM in the following) [4,9,11] one has different scales for each $\alpha_{s}$-term, i.e.

$$
\hat{a}(Q)=a\left(\mu_{1}\right)+r_{1}\left(\mu_{1}\right) a^{2}\left(\mu_{2}\right)+r_{2}\left(\mu_{1}, \mu_{2}\right) a^{3}\left(\mu_{3}\right)
$$

where $\mu_{1}$ is parametrised as $\mu_{1}=\mu_{0} \exp [\theta a(\mu)]$ and $\mu$ as well as $\mu_{3}$ are arbitrary (they will be fixed in higher order approximations but here we simply set them to be the same as $\mu_{2}$ ).

The MBLM scale fixing method is constructed to have $\beta_{2}$ unchanged and instead $\theta$ and $\mu_{2}$ are introduced which gives three $\left(\mu_{1}=\mu_{0} \exp [\theta a(\mu)], \mu_{2}\right.$ and $\left.\beta_{2}\right)$ unphysical parameters instead of the minimal two $\left(\mu\right.$ and $\left.\beta_{2}\right)$. Requiring that the effective charge does not depend on these parameters, to the present order of perturbation theory, gives the explicit $\mu^{-}, \beta_{j^{-}}$ and $\theta$-dependence of the coefficients $r_{i}$,

$$
\begin{aligned}
r_{1}= & r_{1}^{*}+\beta_{0}\left[d^{*}+\ln \frac{\mu_{0}}{Q}\right], \\
r_{2}= & r_{2}^{*}-\frac{\beta_{2}-\beta_{2, \overline{M S}}}{\beta_{0}}+\beta_{1}\left[d^{*}+\ln \frac{\mu_{0}}{Q}\right]+\beta_{0} \theta+ \\
& +\beta_{0}\left[e^{*}+2 r_{1} \ln \frac{\mu_{2}}{Q}\right]+\beta_{0}^{2}\left[f^{*}-\ln ^{2} \frac{\mu_{0}}{Q}\right],
\end{aligned}
$$

where again the integration constants are fixed by assuming that the calculation was made in the $\overline{M S}$ scheme with $\mu=Q$. Comparing with Eqs. (11, 12) we see the effects of having different renormalisation scales and also how the $\theta$-dependence enters. In the MBLM scale fixing all $N_{f}$-dependent terms should be absorbed so that, $r_{1}=r_{1}^{*}$ and $r_{2}=r_{2}^{*}$ just as in the conformal limit scheme. Keeping in mind that $\beta_{2}$ should be unchanged we see that this can be achieved by choosing

\footnotetext{
${ }^{1}$ when two physical observables are related the MBLM (and SBLM) method uses the effective charge $\hat{\beta}_{2}$ of one of the observables which in principle is a measurable quantity.
} 


$$
\begin{aligned}
\beta_{2} & =\beta_{2, \overline{M S}}, \\
\mu_{0} & =Q \exp \left(-d^{*}\right), \\
\theta & =\beta_{0}\left(-f^{*}+\left(d^{*}\right)^{2}\right), \\
\mu_{2} & =Q \exp \left[-e^{*} /\left(2 r_{1}^{*}\right)\right],
\end{aligned}
$$

so that $\mu_{1}=Q \exp \left\{-d^{*}-\beta_{0}\left[f^{*}-\left(d^{*}\right)^{2}\right] a\left(\mu_{2}\right)\right\}$. From Eqs. (20,21) it is also easy to see that one only needs a single renormalisation scale if $\theta$ is chosen appropriately. In this single-scale extension [10] of the BLM scale fixing method (denoted SBLM in the following) one chooses $\mu_{2}=\mu_{1}=\mu_{0} \exp [\theta a(\mu)]$ where $\mu_{0}=Q \exp \left(-d^{*}\right)$ and $\theta=\beta_{0}\left(-f^{*}+\left(d^{*}\right)^{2}\right)-e^{*}+2 r_{1}^{*} d^{*}$.

\section{FIXING THE INITIAL SCHEME WITH A PHYSICAL OBSERVABLE}

Up to now we have assumed that the initial renormalisation scheme (and thereby $\Lambda$ ) is fixed. Now we will show how this can be accomplished using a physical observable so that a unique prediction of another physical observable can be made. As an example we will relate $R$ defined in Eq. (3), to $K$, Bjorken's sum rule for polarized deep-inelastic electroproduction 12].

The effective charge for $R$ is in NNLO given by (in the $\overline{M S}$ scheme),

$$
\hat{a}_{R}=a_{\overline{M S}}\left(1+r_{1} a_{\overline{M S}}+r_{2} a_{\overline{M S}}^{2}\right),
$$

where $r_{1}$ and $r_{2}$ can be obtained from [13,14]. For Bjorken's sum rule, one can also define an effective charge $\hat{a}_{K}$ (using the same normalisation as in 15]),

$$
\begin{aligned}
K & =\int_{0}^{1} d x\left[g_{1}^{e p}\left(x, Q^{2}\right)-g_{1}^{e n}\left(x, Q^{2}\right)\right]= \\
& =\frac{1}{6}\left|\frac{g_{A}}{g_{V}}\right|\left(1-\frac{3}{4} C_{F} \hat{a}_{K}(Q)\right) .
\end{aligned}
$$

In NNLO $\hat{a}_{K}$ is given by,

$$
\hat{a}_{K}=a_{\overline{M S}}\left(1+k_{1} a \overline{M S}+k_{2} a \frac{2}{M S}\right)
$$

where $k_{1}$ and $k_{2}$ have been calculated in [16].

Recognising that the $\overline{M S}$ scheme is only an intermediary scheme suited for calculations we can find a unique relation between the two observables $\hat{a}_{R}$ and $\hat{a}_{K}$. Inverting Eq. (22) for $a_{\overline{M S}}$ and inserting into Eq. (24) gives

$$
\hat{a}_{K}\left(Q_{K}\right)=\hat{a}_{R}\left(Q_{R}\right)\left(1+c_{1} \hat{a}_{R}\left(Q_{R}\right)+c_{2} \hat{a}_{R}^{2}\left(Q_{R}\right)\right)
$$

where now $Q_{R}$ is the renormalisation scale. The coefficients $c_{i}$, which are independent of the intermediate scheme, are given by

$$
c_{1}=-\frac{3}{4} C_{F}-\beta_{0}\left(\frac{7}{4}-2 \zeta_{3}-\ln \frac{Q_{R}}{Q_{K}}\right),
$$




$$
\begin{aligned}
c_{2}= & \frac{9}{16} C_{F}^{2}-\ell-\frac{\beta_{2}-\hat{\beta}_{2, R}}{\beta_{0}}-\beta_{1}\left(\frac{7}{4}-2 \zeta_{3}-\ln \frac{Q_{R}}{Q_{K}}\right)+ \\
& +\beta_{0} C_{F}\left(\frac{523}{144}+\frac{14}{3} \zeta_{3}-10 \zeta_{5}\right)+ \\
& -\beta_{0} N_{C}\left(\frac{13}{36}-\frac{1}{3} \zeta_{3}\right)+\beta_{0} 2 c_{1} \ln \frac{Q_{R}}{Q_{K}}- \\
& -\beta_{0}^{2}\left[-\frac{17}{6}+\left(\frac{35}{3}-8 \zeta_{3}\right) \zeta_{3}-\frac{\pi^{2}}{12}+\ln ^{2} \frac{Q_{R}}{Q_{K}}\right],
\end{aligned}
$$

where $\ell$ is the light-by-light term巳,

$$
\ell=\frac{d^{a b c} d^{a b c}\left(\sum_{i=1}^{N_{f}} e_{i}\right)^{2}}{N_{C} C_{F} \sum_{i=1}^{N_{f}} e_{i}^{2}}\left(\frac{11}{144}-\frac{1}{6} \zeta_{3}\right) .
$$

Hereby all dependence on the $\overline{M S}$ scheme has disappeared. Effectively what we have done is to go from the $\overline{M S}$ scheme to the $R$-scheme, the effective charge scheme for $R$ which is the renormalisation scheme where $\hat{a}_{R}\left(Q_{R}\right)$ has no perturbative corrections, i.e. $\beta_{2}=\hat{\beta}_{2, R}$ and $\ln \left(\mu / \Lambda_{R}\right)=-\hat{r}_{1} / \beta_{0}$ as seen from Eqs. (9,10).

Applying the conformal limit criteria so that the coefficients $c_{1}$ and $c_{2}$ take their conformal limit values, $c_{1}^{*}=-\frac{3}{4} C_{F}=-1$ and $c_{2}^{*}=\frac{9}{16} C_{F}^{2}-\ell=1-\ell$, we get the conformal limit renormalisation scheme parameters $Q_{R}^{*}$ and $\beta_{2}^{*}$,

$$
\begin{aligned}
Q_{R}^{*} & =Q_{K} \exp \left(\frac{7}{4}-2 \zeta_{3}\right), \\
\beta_{2}^{*} & =\hat{\beta}_{2, K}+c_{1}^{*} \beta_{1}+\left(c_{1}^{*}\right)^{2} \beta_{0}-c_{2}^{*} \beta_{0}= \\
& =\hat{\beta}_{2, K}-\beta_{1}+\ell \beta_{0},
\end{aligned}
$$

where $\beta_{2}^{*}$ is obtained from the invariant $\hat{\beta}_{2, K}=\beta_{2}^{*}-c_{1}^{*} \beta_{1}-\left(c_{1}^{*}\right)^{2} \beta_{0}+c_{2}^{*} \beta_{0}$. Eq. (29) has been called a commensurate scale relation [4] since it gives the relation between the renormalisation scales when two physical observables are related. In the same sense one can call Eq. (30) a commensurate $\beta_{2}$ relation since it gives $\beta_{2}$ when two observables are related. The resulting value for $\beta_{2}^{*}$ is an intermediate value between the two effective charge values $\hat{\beta}_{2, K}$ and $\hat{\beta}_{2, R}$. For a general relation between two effective charges, $\hat{a}_{A}=\hat{a}_{B}\left(1+r_{1, A / B} \hat{a}_{B}+r_{2, A / B} \hat{a}_{B}^{2}\right), \beta_{2}^{*}$ depends on the conformal limit values of the coefficients $r_{i, A / B}^{*}$. If $r_{i, A / B}^{*}=0$ then $\beta_{2}^{*}=\hat{\beta}_{2, A}$ and if $r_{i, A / B}^{*}=r_{i, A / B}$ then $\beta_{2}^{*}=\hat{\beta}_{2, B}$. In other words the conformal limit scheme value $\beta_{2}^{*}$ 'interpolates' between the two effective charge values, $\hat{\beta}_{2, A}$ and $\hat{\beta}_{2, B}$, depending on the conformal limit values of the coefficients.

Finally we have the conformal limit result in NNLO,

\footnotetext{
${ }^{2}$ Numerically the light-by-light term is small: $\ell=-0.0376$ for $N_{f}=5, \ell=-0.1653$ for $N_{f}=4$ and $\ell=0$ for $N_{f}=3$. The light-by-light term is not affected by the conformal limit arguments since it is not proportional to $\beta_{0}$.
} 


$$
\hat{a}_{K}\left(Q_{K}\right)=a_{R}^{*}\left(1-a_{R}^{*}+(1-\ell)\left(a_{R}^{*}\right)^{2}\right),
$$

which relates one effective charge $\left(\hat{a}_{K}\right)$ to another one $\left(a_{R}^{*}\right)$ which has been modified in a unique way. This relation resembles the non-perturbative Crewther relation [17], $3 S=K R^{\prime}$, which is derived using conformal and chiral invariance. It relates Adler's anomalous constant $(S)$, Bjorken's sum rule for polarized deep-inelastic electroproduction $(K)$ and the isovector part of $\mathrm{R}\left(R^{\prime}\right)$. According to the no-renormalisation theorem for the axial anomaly [18] one might think [19] that the perturbative corrections to $K$ and $R$ cancel. This is not the case, but instead one finds [19] that the combined corrections are proportional to the $\beta$-function, $\left(1+\hat{a}_{R}\right)\left(1-\hat{a}_{K}\right)-1 \propto \beta(a)$, if the light-by-light term is neglected. The generalised Crewther relation has been studied in more detail in [10].

The modified effective charge, $a_{R}^{*}\left(Q_{R}^{*}, \beta_{2}^{*}\right)$, can be obtained from the third order standard solution to Eq.(6),

$$
\begin{aligned}
a_{R}^{*}\left(Q_{R}^{*}, \beta_{2}^{*}\right)= & \frac{1}{\beta_{0} \ln \left(Q_{R}^{*} / \Lambda_{R}\right)}-\frac{\beta_{1} \ln \ln \left(Q_{R}^{*} / \Lambda_{R}\right)}{\beta_{0}^{3} \ln ^{2}\left(Q_{R}^{*} / \Lambda_{R}\right)}+ \\
& +\frac{\beta_{1}^{2} \ln ^{2} \ln \left(Q_{R}^{*} / \Lambda_{R}\right)-\beta_{1}^{2} \ln \ln \left(Q_{R}^{*} / \Lambda_{R}\right)+\beta_{2}^{*} \beta_{0}-\beta_{1}^{2}}{\beta_{0}^{5} \ln ^{3}\left(Q_{R}^{*} / \Lambda_{R}\right)},
\end{aligned}
$$

which is valid for $\ln \left(Q_{R}^{*} / \Lambda_{R}\right) \gg 1$. The value of $\Lambda_{R}$ should be determined by experiment from $\hat{a}_{R}\left(Q_{R}\right)$ with $\beta_{2}=\hat{\beta}_{2, R}$ and $Q_{R}=\sqrt{s}$ using the same solution for $\alpha_{s}$. (The definition of $\Lambda$ depends on which solution that is used but sticking to one definition/solution this presents no problem.) In other words the effective charge $\hat{a}_{R}\left(Q_{R}\right)$ gives an experimentally measurable $\Lambda_{R}$ and a well defined starting renormalisation scheme which is then modified into the conformal limit scheme where the scheme parameters are given by $Q_{R}^{*}$ and $\beta_{2}^{*}$.

\section{DISCUSSION}

Fig. [1(a,b) illustrates the renormalisation scheme dependence of $\hat{a}_{K}\left(Q_{K}=50 \mathrm{GeV}\right)$ as given by Eq. (25) using the standard solution, Eq. (32), for $a_{R}$ with $\Lambda_{R}^{(5)}=502 \mathrm{MeV}$. We see that for not too small renormalisation scales the $\beta_{2}$-dependence dominates whereas for smaller scales both the scale dependence and $\beta_{2}$-dependence is quite strong. Since the renormalisation scheme dependence is parametrised by the renormalisation scale $Q_{R}$ and $\beta_{2}$ when $\Lambda_{R}$ is kept fixed, the whole space of schemes should in principle be obtained by varying $Q_{R}$ and $\beta_{2}$. However, this does not take into account the region of validity for Eq. (32). For too small renormalisation scales $Q_{R}$ or too large $\beta_{2}$ this solution is no longer valid.

To be self-consistent, one should also take into account that Eq. (6) has to make sense perturbatively. In other words, if $\left|\beta_{2}\right| a_{R} \geq \beta_{1}$ then we are no longer in the perturbative regime where the contribution from consecutive terms are smaller then the preceding ones and therefore the perturbative expansion breaks down. The lines $\beta_{2}= \pm \beta_{1} / a_{R} \simeq \pm \beta_{1} \beta_{0} \ln \left(Q_{R} / \Lambda_{R}\right)$ which indicates where this happens are shown in Fig. 四(b). These lines also indicates where the solution given by Eq. (32) is no longer valid and the numerical results should therefore

not be trusted in that region. The conformal limit scheme fixing method (CLSF) and the SBLM scale fixing method are indicated in Fig. 目(b) together with some other well-known 

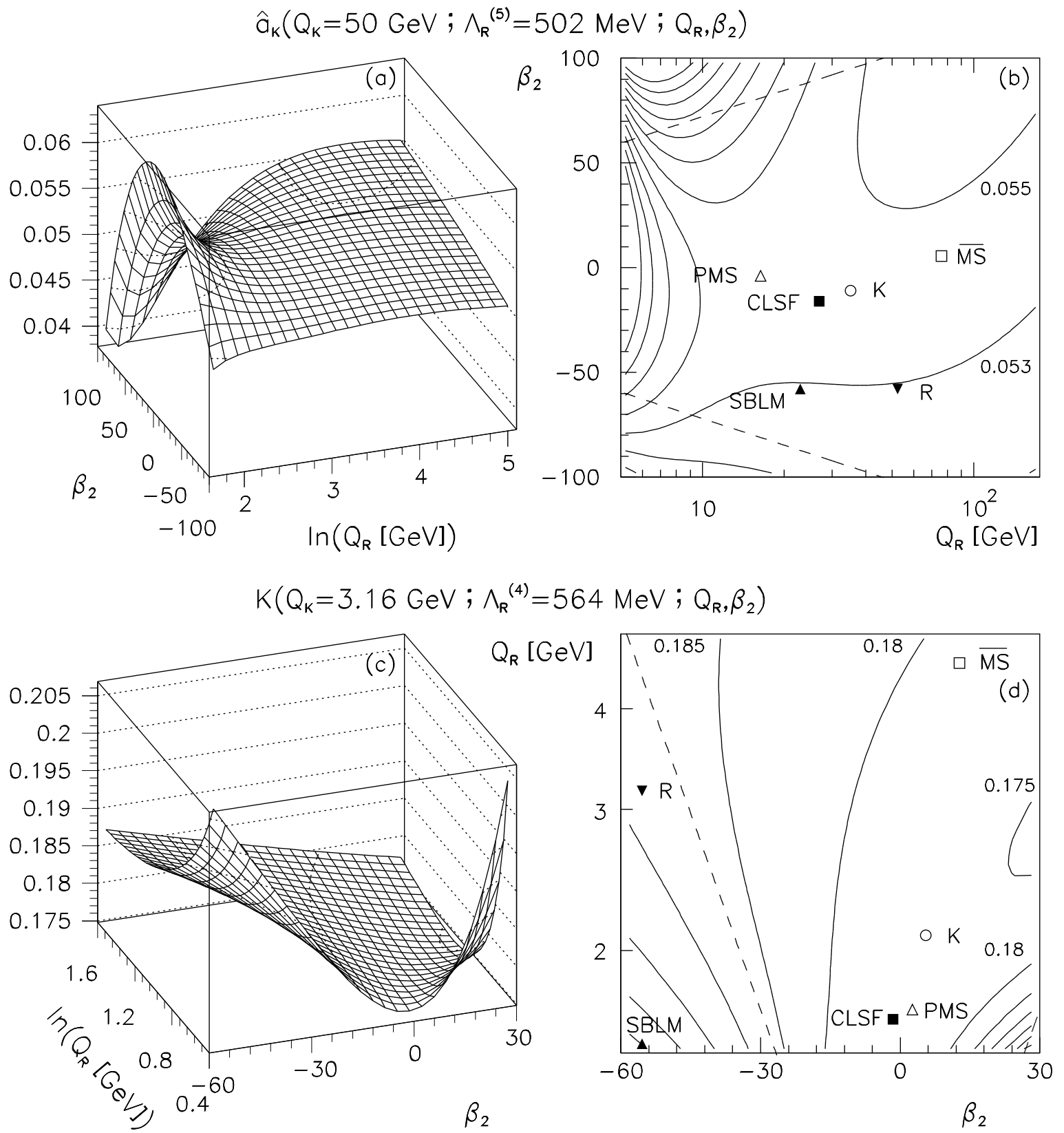

FIG. 1. Renormalisation scheme dependence of $\hat{a}_{K}\left(Q_{K}=50 \mathrm{GeV}\right)$ in $(\mathrm{a}, \mathrm{b})$ and Bjorken's sum rule, $K\left(Q_{K}=3.16 \mathrm{GeV}\right)$ in $(\mathrm{c}, \mathrm{d})$; shown as surface plots $(\mathrm{a}, \mathrm{c})$ and contour plots $(\mathrm{b}, \mathrm{d})$. Note that $\Lambda$ has been kept fixed so that all the renormalisation scheme dependence is given by the renormalisation scale $Q_{R}$ and $\beta_{2}$. Some well-known scheme and scale choices are marked in (b) and (d) for reference and the corresponding numerical values are given in Table 1 and II respectively. The dashed lines indicates the limit of the perturbative regime, $\left|\beta_{2}\right| \leq \beta_{1} / a_{R} \simeq \beta_{1} \beta_{0} \ln \left(Q_{R} / \Lambda_{R}\right)$ as explained in the text. In addition to the scheme dependence there is also a experimental uncertainty from the value of $\Lambda_{R}$. 
schemes like the $\overline{M S}$ scheme, the 'Principle of Minimum Sensitivity' (PMS) and the effective charge schemes (ECH) for $R$ and $K$.

Conceptually the PMS and ECH schemes are different from the conformal limit schemes in that they prescribe a unique scheme for each observable instead of giving schemes for relations between observables. It should also be noted that the PMS and ECH schemes sometimes gives renormalisation scales which are difficult to interpret physically. For instance in jet-production, both in $e^{+} e^{-}$[20] and DIS [21], the resulting renormalisation scales grow when the typical jet-mass $\left(y_{c u t} W^{2}\right)$ is decreased which is counter-intuitive. In addition the PMS prescription depends on the intermediate scheme. For example, applying the PMS prescription to two observables given in the $\overline{M S}$ scheme separately and then relating them gives a different result compared to if the observables are first related so that the dependence on the $\overline{M S}$ scheme is removed and then the PMS prescription is applied.

For reference, the numerical values of $\beta_{2}, Q_{R}, \hat{a}_{K}$ and Bjorken's sum rule $K$ in the different schemes are given in Table \& (together with the MBLM method which has two renormalisation scales). Comparing the conformal limit scheme fixing (CLSF) with the SBLM and MBLM scale fixing methods in Table If we see that even though the coefficients $c_{i}$ are the same, the scales, $\beta_{2}$ and the resulting effective charge $\hat{a}_{K}$ are different. This shows the importance of not only taking the commensurate scale relation into account as in the SBLM and MBLM methods but also the commensurate $\beta_{2}$ relation as in CLSF.

TABLE I. Numerical values of $\beta_{2}, Q_{R}, \hat{a}_{K}$ and Bjorken's sum rule $K$ in different schemes for $Q_{K}=50 \mathrm{GeV}$ and $\Lambda_{R}^{(5)}=502 \mathrm{MeV}\left(\Lambda\right.$ is kept fixed so that the scheme dependence is given by $\beta_{2}$ and $\left.Q_{R}\right)$. Note that there are two scales for the MBLM method given as $Q_{R, 1}\left(Q_{R, 2}\right)$.

\begin{tabular}{ccccc}
\hline \hline Scheme & $\beta_{2}$ & $Q_{R}[\mathrm{GeV}]$ & $\hat{a}_{K}$ & $K$ \\
\hline CLSF & -15.98 & 26.00 & 0.5404 & 0.1982 \\
SBLM & -57.86 & 22.18 & 0.5271 & 0.1985 \\
MBLM & -57.86 & $22.17(25.02)$ & 0.5290 & 0.1985 \\
$R$ & -57.86 & 50.00 & 0.5291 & 0.1985 \\
$K$ & -11.00 & 33.74 & 0.5421 & 0.1982 \\
$\overline{M S}$ & 5.65 & 72.22 & 0.5444 & 0.1981 \\
PMS & -3.98 & 15.88 & 0.5403 & 0.1982 \\
\hline \hline
\end{tabular}

From Fig. 1(b) we also see that the CLSF point is closer to the saddle-point (PMS) than the SBLM point, which means that the scheme dependence is smaller in the CLSF point. One might also worry that the SBLM and MBLM scale fixing methods are too close to the line $\beta_{2}= \pm \beta_{1} \beta_{0} \ln \left(Q_{R} / \Lambda_{R}\right)$ where the perturbative expansion for the effective charge $\hat{a}_{R}$ breaks down. Note that both in Fig. 1 and in Table $\mathbf{I}$ (and II) the renormalisation scales are related to $\Lambda_{R}$. This means that for example in the $\overline{M S}$ scheme where one normally would use $\mu=Q_{K}$ as the renormalisation scale for $\Lambda=\Lambda \overline{M S}$, the scale becomes $Q_{K} \exp \left(r_{1, \overline{M S}} / \beta_{0}\right)$ for $\Lambda=\Lambda_{R}$ (compare with Eq. (9)) .

As a concrete example of the conformal limit scheme fixing method we will use the global analysis of $R_{e+e-}$ data in the range $2.64<Q_{R}<52 \mathrm{GeV}$ by Marshall [22] to calculate the Bjorken sum rule $K$ at $Q_{K}=3.16=\sqrt{10} \mathrm{GeV}$, which can be compared with the SMC measurement, $K=\Gamma_{1}^{p}-\Gamma_{1}^{n}=0.199 \pm 0.038$ [15]. The result of the analysis in [22] is a global 
fit taking both electroweak and QCD corrections into account, $R_{e+e-}=R_{\text {Born }}^{\gamma, Z}\left(1+\hat{a}_{R}\right)$, and numerical values for $R_{Q}=1+\hat{a}_{R}$ are given for some distinct energies. In the following we have used the value $R_{Q}=1.0463 \pm 0.0044$ for $Q_{R}=59.2 \mathrm{GeV}$. The reasons for picking this particular energy are that we want to have a large scale $Q_{R}$ where the standard solution, Eq. (32), is a good approximation (especially since $\hat{\beta}_{2, R}$ is so large) and at the same time we do not want to extrapolate the experimental result too much outside the measured range.

Following the prescription given above for determining $\Lambda_{R}^{(5)}$ we get,

$$
\Lambda_{R}^{(5)}=502_{-225}^{+326} \mathrm{MeV},
$$

from $\hat{a}_{R}=0.0463 \pm 0.0044$ using Eq. (32) with $\hat{\beta}_{2, R}=-57.9$ and $Q_{R}=59.2 \mathrm{GeV}$. To be able to compare with the SMC measurement we also need $\Lambda_{R}^{(4)}$. This is obtained by matching $a_{R}^{*}$ numerically at the flavour threshold, $Q_{R}=m_{b}=5 \mathrm{GeV}$, for $N_{f}=4$ and 5 with $\beta_{2}=\beta_{2}^{*}$ which gives,

$$
\Lambda_{R}^{(4)}=564_{-224}^{+282} \mathrm{MeV} .
$$

The conformal limit renormalisation scheme parameters are then obtained from Eqs. (29) and (30), $Q_{R}^{*}=1.64 \mathrm{GeV}$ and $\beta_{2}^{*}=-1.56$, and together with the conformal limit coefficients $c_{1}^{*}=-1, c_{2}^{*}=1.165$ and $\Lambda_{R}^{(4)}$ this gives $\hat{a}_{K}=0.159_{-0.048}^{+0.139}$. Finally the conformal limit result for Bjorken's sum rule given by Eq. (23) becomes

$$
K=0.176_{+0.010}^{-0.029},
$$

where $\left|g_{A} / g_{V}\right|=1.2573 \pm 0.0028$ [23] has been used and the error comes from the uncertainty in $R_{Q}$. This is in good agreement with the experimental value $K=0.199 \pm 0.038$ measured by SMC. To be able to make a more challenging test of the conformal limit scheme arguments one would need much more precise measurements of both $R_{e+e-}$ and $K$.

For illustration we also show the renormalisation scheme dependence of Bjorken's sum rule in Fig. 目 $(\mathrm{c}, \mathrm{d})$ and the numerical values of $\beta_{2}$ and $Q_{R}$ for the specific schemes indicated in Fig. 1(d) are given in Table \ together with the resulting values for $\hat{a}_{K}$ and Bjorken's sum rule $K$. Comparing with Fig. 1 1 (a,b) we see that the scheme dependence is much stronger which is also expected since we are at a much smaller $Q_{K}$. We also see that the perturbative regime indicated by the dashed line in Fig. 1 $1(d)$ is narrower than in Fig. 1(b) and in fact both the SBLM and MBLM methods as well as the $R$-scheme are outside the perturbative regime. Therefore the numerical results given for these schemes should not be trusted. However, one must keep in mind that the SBLM and MBLM scale fixing methods advocates the use of a physical measurement of $\hat{a}_{R}$ at this scale and in this way the problem with the validity of the solution used for $\alpha_{s}$ never enters. Even so, Fig. 1 $(\mathrm{c}, \mathrm{d})$ illustrates clearly that there is a strong renormalisation scheme dependence for Bjorken's sum rule at this scale which should be taken into account when comparing the experimental result with theoretical expectations.

\section{SUMMARY AND CONCLUSIONS}

In summary we have shown that it is possible to generalise the conformal limit arguments of the BLM method to NNLO to fix the renormalisation scheme, i.e. both the renormalisation scale and $\beta_{2}$, when two observables are related. In this way all signs of scale breaking, 
TABLE II. Numerical values of $\beta_{2}, Q_{R}, \hat{a}_{K}$ and Bjorken's sum rule $K$ in different schemes for $Q_{K}=3.16 \mathrm{GeV}$ and $\Lambda_{R}^{(4)}=564 \mathrm{MeV}$ ( $\Lambda$ is kept fixed so that the scheme dependence is given by $\beta_{2}$ and $\left.Q_{R}\right)$. Note that there are two scales for the MBLM method given as $Q_{R, 1}\left(Q_{R, 2}\right)$.

\begin{tabular}{ccclc}
\hline \hline Scheme & $\beta_{2}$ & $Q_{R}[\mathrm{GeV}]$ & $\hat{a}_{K}$ & $K$ \\
\hline CLSF & -1.56 & 1.64 & 0.159 & 0.176 \\
SBLM & -55.46 & 1.53 & 0.0229 & 0.205 \\
MBLM & -55.46 & $1.49(1.58)$ & 0.0126 & 0.207 \\
$R$ & -55.46 & 3.16 & 0.100 & 0.189 \\
$K$ & 5.54 & 2.09 & 0.160 & 0.176 \\
$M S$ & 12.70 & 4.56 & 0.147 & 0.179 \\
PMS & 2.62 & 1.69 & 0.160 & 0.176 \\
\hline \hline
\end{tabular}

i.e. $\beta \neq 0$, is confined into the running of the coupling and the coefficients in the perturbative series have their conformal limit values. We have also shown (contrary to [9]) that this prescription for making the coefficients have their conformal limit values is unique. Comparing with earlier extensions of the BLM method to NNLO they only fix the scale using $\beta_{2}$ from the effective charge. This means, that the conformal limit scheme fixing gives both a so called commensurate scale relation as well as a commensurate $\beta_{2}$ relation which, in a unique way, specifies how $\beta_{2}$ should be modified when two physical observables are related to each other.

Applying the conformal limit scheme fixing method to the relation between Bjorken's sum rule $K$ and $R_{e+e-}$ gives a simple relation between the two. Using the effective charge value $\hat{a}_{R}=0.0463 \pm 0.0044$ for $Q_{R}=59.2 \mathrm{GeV}$ from a global analysis of $R_{e+e-}$ data gives $K=0.176_{+0.010}^{-0.029}$ for $Q_{K}^{2}=10 \mathrm{GeV}^{2}$ where the error comes from the experimental uncertainty in $\hat{a}_{R}$. Assessing a theoretical error is much more complicated. The theoretical uncertainty is illustrated by the renormalisation scheme dependence which is shown to be quite large even though it can be reduced by requiring the scheme to belong to the perturbative regime. Still, the problem of quantifying the theoretical error remains to be solved. However, comparing with the experimentally measured $K=0.199 \pm 0.038$ the agreement is good and theoretical uncertainties are still smaller than the experimental ones.

\section{ACKNOWLEDGMENTS}

I would like to thank Stan Brodsky and Gunnar Ingelman for useful discussions on the subject of this paper. I also want to thank Hung Jung Lu for helpful remarks on the MBLM scale fixing method. 


\section{REFERENCES}

[1] G. Grunberg, Phys. Lett. 95B, 70 (1980); Phys. Rev. D 29, 2315 (1984).

[2] P. M. Stevenson, Phys. Rev. D 23, 2916 (1981).

[3] S. J. Brodsky, G. P. Lepage and P. B. Mackenzie, Phys. Rev. D 28, 228 (1983).

[4] S. J. Brodsky and H. J. Lu, Phys. Rev. D 51, 3652 (1995).

[5] G. P. Lepage and P. B. Mackenzie, Phys. Rev. D 48, 2250 (1993).

[6] M. Neubert, Phys. Rev. D 51, 5924 (1995).

[7] P. Ball, M. Beneke and V. M. Braun, Nucl. Phys. B 452, 563 (1995).

[8] J. Rathsman, TSL/ISV preprint in preparation.

[9] G. Grunberg and A. L. Kataev, Phys. Lett. B279, 352 (1992).

[10] S. J. Brodsky, G. T. Gabadadze, A. L. Kataev and H. J. Lu, DESY preprint 95-245, e-Print Archive hep-ph/9512367.

[11] L. R. Surguladze and M. A. Samuel, Phys. Lett. B309 , 157 (1993).

[12] J. D. Bjorken, Phys. Rev. 148, 1467 (1966); Phys. Rev. D 1, 1376 (1970).

[13] S. G. Gorishny, A. L. Kataev and S. A. Larin, Phys. Lett. B259, 144 (1991).

[14] L. R. Surguladze and M. A. Samuel, Phys. Rev. Lett. 66, 560 (1991); 66, 2416(E) (1991).

[15] SMC Coll. (D. Adams et. al.), Phys. Lett. B357, 248 (1995).

[16] S. A. Larin and J. A. M. Vermaseren, Phys. Lett. B259, 345 (1991).

[17] R. J. Crewther, Phys. Rev. Lett. 28, 1421 (1972).

[18] S. L. Adler and W. A. Bardeen, Phys. Rev. 182, 1517 (1969).

[19] D. J. Broadhurst and A. L. Kataev, Phys. Lett. B315, 179 (1993).

[20] G. Kramer, B. Lampe, Z. Phys. A 339, 189 (1991).

[21] G. Ingelman and J. Rathsman, Z. Phys. C 63, 589 (1994).

[22] R. Marshall, Z. Phys. C 43, 595 (1989).

[23] L. Montanet et al., Phys. Rev. D 50, 1173 (1994). 